\title{
EyePhone Technology: A Smart Wearable Device
}

\author{
K. G. Kharade ${ }^{\text {1 }}$, S.K.Kharade ${ }^{\text {b }, ~ S . ~ R . ~ G h a t a g e ~}{ }^{c}$, V.S.Kumbhar ${ }^{\mathrm{a}}$, \\ T. Nandana Ravishankard ${ }^{\text {, K.Vengatesan }}{ }^{\mathrm{e}}$ \\ ${ }^{a}$ Department of Computer Science, Shivaji University, Kolhapur, MH \\ ${ }^{b}$ Department of Mathematics, Shivaji University, Kolhapur, $M H$ \\ ${ }^{c}$ Gopal Krishna Gokhale College, Kolhapur, $M H$ \\ ${ }^{d}$ Department of Computer Science \& Engineering, Vel Tech High Tech, Avadi, Chennai, TN \\ ${ }^{e}$ Department of Computer Engineering, Sanjivani College of Engineering, Kopargaon, MH
}

\begin{abstract}
In the past few decades, wearable sensors and devices have evolved into central technologies that have significantly impacted next-generation healthcare solutions in the previous decade. It is an age of incredibly cut-throat competition, even the youth. This technology is Hand-free, eyephone-operated telephone technology. It measures where the user's eyes are located on the phone's display employing a camera attached to the device. The typical work the eye bones assigned to eye tracking, eye blinking, detecting, etc., by way of eye-tracking, users can take care of their email, calendar, phone, etc. The leading technologies in use are a Human-Computer Interface (HCI) and a Human Phone Interface (HPI). It helps those with disabilities greatly. The IT Index is an expanded form of HCI. The use of mobile devices like smartphones and tablets is on the rise, and, to some extent, it could displace the use of desktops and laptops. Human-computer interaction is interested in the interaction between people and the computer system and between software and applications. Our focus is on this revolutionary new type of technology called the eye-phone.
\end{abstract}

Keywords. Eye Phone, E-Healthcare, HCI, HPI, Mobile Sensing Systems, Mobile Phones

\section{Introduction}

A new generation of smartphones has revolutionized touch screens, gyroscopic, 3D cameras, and other technology advancements. The software change and improved hardware performance enable a new way of employing these devices and techniques. Some recent research projects show how communication with mobile devices enhanced the creative process. An important avenue of communication has been opening up to the blind or visually impaired [2]. With the Eye Phone, you can tweet and make phone calls. The latest development of which is the widespread use of touch screens. With this touch screen, it's much easier to interact with mobile phones. It can locate and map the user's eyes on the screen, using a wink to establish the association with any application. At no time does the user have to be touching the phone or the phone display to use the device. With constrained form factors such as smartphones, researchers and vendors constantly look for new ways to cut down on the amount of

\footnotetext{
${ }^{1}$ K. G. Kharade, Department of Computer Science, Shivaji University, Kolhapur, India Email: kgk_csd@unishivaji.ac.in
} 
effort required to access applications [3]. The user can use it as a phone, and it functions as a surveillance camera as well.

The current EyePhone system can follow a user's eye, and output images can be controlled using the phone's built-in camera [4]. The HPI can be used in a hands-free manner, as it is used in facial recognition.

Additionally, we believe Eyephone technology is a better alternative to voice activation systems in quiet locations. All you need is a front camera to use the phone is one of these. Today's smartphones have a front-facing camera; expect to see even more on future models [18]. Human-computer Interaction (HCI) is a multidisciplinary field of study focusing on the design of computer technology and, in particular, the interaction between humans (the users) and computers [5]. While initially concerned with computers, HCI has expanded to cover almost all forms of information technology design. An HCI in which users can control their phones with their eyes or faces while exercising and developing a proof-of-concept prototype is done using an Android phone fitted with an EEG headset and an eye tracker [19]. Each functional module can be individually launched and switched; it is possible to achieve a "no hands" environment. To show that minds and intentions on mobile devices can be accurately controlled, improve our society's lives and health. Although this paper demonstrates several laboratory problems, it lays the groundwork for further studies on HCI in the outside world [1].

\section{Literature Review}

(Sannidhan, 2018) identified that there are numerous academic and non-profit uses for computer vision research. Multiple technologies are currently available on the market that keeps track of drivers' minds and helps prevent similar incidents from occurring in daily life. Increasing road traffic has brought about a rising number of road accidents every day [20]. (Saba, 2017) found, how to ease human-phone interaction (HPI). From touch-sensitive screens to voice activation systems to EyePhones that incorporate eyetracking software and algorithms, Biometric Goggles that utilizes eye tracking and biometric algorithms will be remarkable and significant to numerous customers. In noisy environments, the overall performance of voice control systems is lessened [4]. Very few researches have been carried out on this topic. Implementation of this technology is costly and can not be affordable by regular users.

\section{Proposed Method}

During this research, we have gone through various papers in this field. EyePhone technology has enormous advantages and can be integrated with many areas. Such areas cover fields like engineering and the medical domain. We can incorporate EyePhone technology to provide value addition to the existing applications.

\section{Hardware Challenges}

As opposed to HCI applications, any HPI implementation should not rely on any external hardware. Asking people to carry or wear additional hardware to use their phones might reduce the technology's penetration. Moreover, state-of-the-art HCI hardware such as glass-mounted cameras or dedicated helmets is not yet small enough to be conformably worn for long periods by people [6]. Any HPI application should 
rely as much as possible on just the phone's onboard sensors. Over the last decade, noninvasive electroencephalogram (EEG) BCIs have greatly improved. But here are some challenges that remain, like the non-stationary and low signal-to-noise ratio of the EEG. It is imperative to consider optimizing both the individual parts of BCIs and their interrelationship to achieve the greatest possible bandwidth. In other words, using concepts from human-computer interaction (HCI) methods in HCI and neuroscience should be combined [19]. One of the primary stumbling blocks in broader adoption is the lack of friendly interfaces and GUIs for patients and medical staff. Heterogeneous sensors can also mean multiple sensor types, including wearable, smartphones, and IoT sensors that allow monitoring to be quick and patient-friendly [17].

\section{Eye Phone Design}

The eye phone algorithmic design breaks down into the following pipeline phases:

1. Eye detection phase

2. Open eye template creation phase

3. Eye-tracking phase

4. Blink detection phase

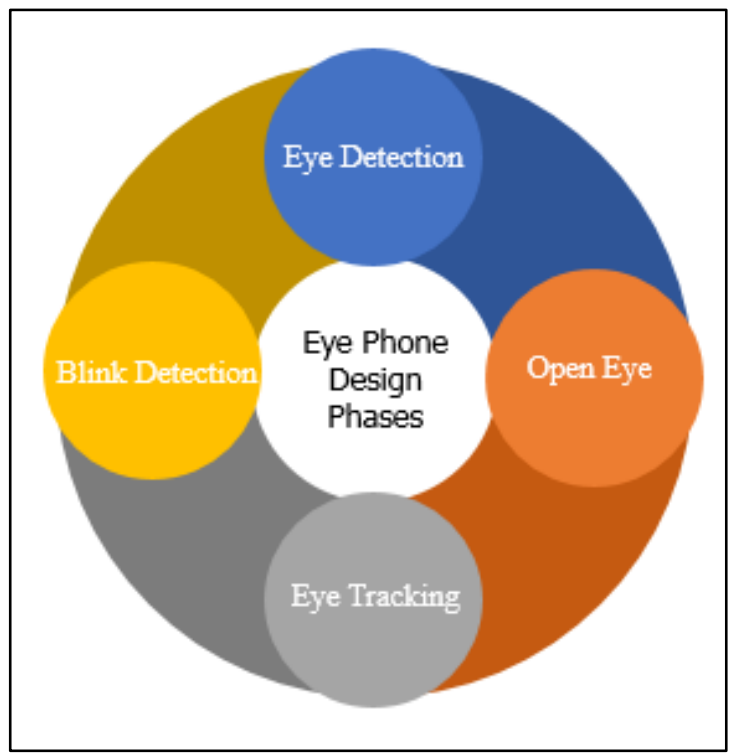

Fig. 1: Eye Phone Design Phases

a) Eye Detection Phase: By applying a motion analysis technique that operates on consecutive frames, this phase consists of finding the contour(outline) of the eyes. The left and right eye contours identify the eye pair. While the original algorithm identifies the eye pair with almost no error when running on a desktop computer with a fixed camera [7].

b) Open Eye Template Creation: While the author is adopting an online open eye template creation by extracting the template every time the eye pair is lost, the eye phone does not rely on the same strategy. Compared to a desktop 
machine, the reduced computation speed and the restricted battery requirements imposed by the N810 dictate a different approach. Instead, an eye phone creates a user's open eye template once at the beginning when a person uses a system for the first time using the eye detection algorithm described above [8].

c) Eye Tracking: The eye-tracking algorithm is based on template matching. The template matching function calculates a correlations core between the open eye template created the first time the application uses and a search window. The search window is limited to twice the box's size, enclosing the eye to reduce the time template matching function's computation time and save resources[9].

d) Blink Detection: To detect blinks, we apply the thresholding (beginning) technique for the normalized correlation coefficient returned by the template matching function as suggested.

\section{Applications of Eye Phone}

EyePhone technology can be integrated with many technologies. Some of the applications of this technology are;

a) It is possible to use one's eyes to detect if one is sleepy and steer clear of the latter.

b) The EyePhone may be able to gauge drowsiness and inattention levels in drivers. While the industry is creating vehicles that monitor drivers' sleep and alertness, the EyePhone can apply to nearly any vehicle type.

c) It is a paradoxical notion to consider virtual reality as part of the game in that it emulates reality while distancing people from it [10].

d) Consumers will buy a higher quality product when they think it is essential.

e) Brands, marketing, offering product placement as a service

f) Due to physical infirmity, especially partial blindness, however, the phone is most often used to guide the eye. can be used while you are walking

g) If the problem lies outside the vision, do an MRI or CT scan, allowing inside eye examination for various medical issues, dental, and surgical issues [11].

\section{Eye-Phone Apps in Healthcare}

Providing healthcare in most developing countries remains a significant problem for healthcare providers in remote areas. Here are some apps where this technology is available;

a) Peek: Portable Eye Examination Kit (Peek) is a community health care worker-focused mobile app designed to enable them to do their work anywhere. The application can scan the eye for various ailments, including diseases, visual acuity, visual field, and color vision [16]. Indicating Peek may detect different types of blindness, such as glaucoma, macular degeneration, macular and nerve tumors, diabetic retinopathy, and brain hemorrhage. With only basic training, the app can conduct a comprehensive analysis. Hence, it is inexpensive, fragile, and expensive and does not require a constant power supply. In addition, it is often portable and effortless to use [12]. 
b) D-EYE: It's a window into the human mind's world. It's an innovative concept in eye examinations that will make them available to the general public, team up with telemedicine, and increase healthcare access.

c) Wearable EEG headset: It is a design with a graphical window that receives continuous EEG data from the headset and motion tracking. It is also possible to implement a cursor to simulate movement on the screen and face recognition [13].

d) Mindo: Mindo monitors every change in the driver's mental activity as it happens. With Java as a mobile application for online analysis, the system processes the brain wave recordings to derive the level of drivers of vigilance. A neuro-backpack system incorporating electroencephalography (EEG) and transcranial electrical stimulation (TES) is now available. Independent component analysis (ICA), neuro-system architecture incorporating the FFT and support vector machine (SVM) [14].

e) Tele rehabilitation Device: a novel device intended for the requirements of patients with rheumatoid arthritis. It has several sensors that can be used either in a clinic for functional hand evaluation or by the patient at home for therapy, with a PC integration. In the second case, the device assesses the patient's performance as he follows the session guidance, using a GSM/GPRS-based connection to collect statistics for future analysis [18].

f) Mobicare Cardio Monitoring System: It is a predictive virtual instrument that consists of a cellular phone with real-time Q-T onset detection algorithms, Q on-time, T out-time (mobile ECG), a Web-enabled ECG sensor, a patient's database, and a web-rich server was recently introduced. Under this arrangement, MobiECGPRS+ sends the abnormally detected signal to hospitals to not burden the doctor [6].

g) WBSN based e-healthcare: The Wireless Body Sensor Network-based ehealthcare Systems is wearable, wireless communication, multi-sensor data fusion is utilized. The BSN continuously monitors important network parameters, such as physiological data security [15].

\section{Conclusion}

We presented the eye-phone technology and focused on using it in practice, and assessed its usefulness. For a mobile interface, the eyephone relies on eye-tracking and blinks. Our preliminary findings show that using the eyephone to develop mobile applications in a hands-free manner is exciting. The growth in e-health, driven by wireless and sensor technologies, which have accelerated e-healthcare. As e-health architecture has developed, it has become more lightweight, compact, and cheap, with smartphone's help. In this paper, we have focused on eye-phone technology, its design, and its application. It also focuses on how it is helpful in e-health care. Finally, it also focuses on its challenges before such technologies.

\section{References}

[1] English, E., Hung, A., Kesten, E., Latulipe, D., \& Jin, Z. (2013). EyePhone: A Mobile EOG-based Human-Computer Interface for Assistive Healthcare. 6th Annual International IEEE EMBS Conference on Neural Engineering (pp. 105-108). San Diego, California: IEEE. 
[2] Miluzzo, E., Wang, T., \& Campbell, A. T. (2010). EyePhone: Activating mobile phones with your eyes. Proceedings of the Second ACM SIGCOMM Workshop on Networking, Systems, and Applications on Mobile Handhelds - MobiHeld '10, 15. https://doi.org/10.1145/1851322.1851328

[3] Schleicher, R., Westermann, T., \& Reichmuth, R. (2014). Mobile Human-Computer Interaction. In S. Möller \& A. Raake (Eds.), Quality of Experience (pp. 339-349). Springer International Publishing. https://doi.org/10.1007/978-3-319-02681-7_23

[4] SSM College of Engineering \& Technology, Kashmir, India, \& Paul, Z. M. (2017). EYE PHONEHAND FREE MOBILE CONTROL: A REVIEW. International Journal of Advanced Research in Computer Science, 8(7), 52-54. https://doi.org/10.26483/ijarcs.v8i7.4226

[5] Understanding Mobile Human-Computer Interaction. (2005). Elsevier. https://doi.org/10.1016/B978-07506-6352-6.X5000-2

[6] Kher, R. K. (2016). Mobile and E-Healthcare: Recent Trends and Future Directions. Journal of Health \& Medical Economics , 2-10.

[7] Dongale, T. D., Kharade, K. G., Naik, G. M., \& Kamat, R. K. (2017). Artificial Neural Network Modeling of NixMnxOx based Thermistor for Predicative Synthesis and Characterization. Journal of Nano And Electronic Physics, 03042(1)-03042(4).

[8] Katkar, S. V., Kharade, K. G., \& Kamat, R. K. (2017). Role of WhatsApp in Farmer's Welfare. National Conference on Rural Livelihoods Models for Sustainable Development organized by Yashvantrao Chavan School of Rural Development, Shivaji University, Kolhapur.

[9] Katkar, S. V., Kharade, K. G., Kharade, S. K., \& Kamat, R. K. (2020). An Intelligent Way of Modeling and Simulation of WO3 for Supercapacitor. In N. Thapa, Recent Studies in Mathematics and Computer Science (pp. 109-117). Hooghly, West Bengal, India: Book Publisher International.

[10] Potharaju, S. P., Sreedevi, M., \& Amiripalli, S. S. (2019). An Ensemble Feature Selection Framework of Sonar Targets Using Symmetrical Uncertainty and Multi-Layer Perceptron (SU-MLP). In Cognitive Informatics and Soft Computing (pp. 247-256). Springer, Singapore.

[11] Kharade, K. G., Kamat, R. K., \& Kharade, S. K. (2017). Utilization of Copy Logger Software Package to Protect Sensitive Data from Malicious Individuals. National conference on "Innovative Trends in Electronics and Allied Technology (ITEAT-2017)" organized by Yashvantrao Chavan Institute of Science, Satara, (p. 19).

[12] Kharade, K. G., Kamat, R. K., Kharade, S. K., \& Katkar, S. V. (2019). Automation of Paper Setting Process to Improve Effectiveness of The Examination System of The University. Journal of Emerging Technologies and Innovative Research, 490-493.

[13] Kharade, K. G., Kamat, R. K., Mudholkar, R. R., \& Kharade, S. K. (2018). Removable Drive Blocker Application for Virus Detection. International Journal of Research Culture Society, 233-234.

[14] Kharade, K. G., Kharade, S. K., \& Kumbhar, V. S. (2018). Impact of Digital India on Various Sectors. Indian Journal of Innovation in Management and Excellence In Research (IJIMER), 37-40.

[15] Kharade, K. G., Kharade, S. K., \& Oza, K. S. (2018). Security Threats In E-commerce. Indian Journal of Innovation In Management And Excellence In Research (IJIMER), 14-17.

[16] Patil, B. P., Kharade, K. G., \&Kamat, R. K. (2020). Investigation on Data Security Threats \& Solutions. International Journal of Innovative Science and Research Technology, 5(1), 79-83.

[17] Rao, G., Kumari, K., Shankar, D., \& Kharade, K. G. (2021). A comparative study of augmented realitybased head-worn display devices. Materials Today: Proceedings., 1-9.

[18] Potharaju, S. P., \& Sreedevi, M. (2018). A novel cluster of quarter feature selection based on symmetrical uncertainty. Gazi University Journal of Science, 31(2), 456-470.

[19] Scherer, R., Josef Faller, D. B., Friedrich, E. V., Pröll, M., Allison, B., \& Müller-Putz, G. (2012). Brain-computer interfacing: more than the sum of its parts. Soft Computing, 317-331..

[20] M S, Sannidhan \& Aithal, Sunil Kumar \& Bhandary, Abhir. (2019). A Comprehensive Review On Various State Of Art Techniques For Eye Blink Detection. 